\title{
Vom Risiko zum Sicherheitskonzept
}

\section{Bewährte Methoden und Werkzeuge}

Risiko ist als das Produkt aus Eintrittshäufigkeit und Schadensausmaß definiert. Dabei werden drei Bereiche unterschieden: ein nicht akzeptabler Bereich, ein tolerierbarer Bereich und ein allgemein akzeptabler Bereich. Vom Risiko kommt man zum Sicherheitskonzept, indem man Schadensszenarien identifiziert und bewertet. Zur Bewertung der Szenarien stehen verschiedene Werkzeuge zur Verfügung. Dies sind deterministische Methoden ohne Risikobezug, der Risikograph, die Layers-of-Protection-Analyse (LOPA), die Risikomatrix und die quantitative Risikoanalyse. In diesem Beitrag wird der Weg vom identifizierten Risiko zum Sicherheitskonzept vorgestellt.

SCHLAGWÖRTER Risiko/Sicherheitskonzept

\section{From Risk to Safety Concept -}

Best Practice Methods and Tools

Risk is defined as the product of frequency of occurrence and severity. Three areas are distinguished: an area being not acceptable, a tolerable area, and a commonly acceptable area. One gets from risk to the safety concept by identifying and assessing hazard scenarios. Several tools are available for the assessment of the scenarios. These include deterministic methodologies without risk consideration, the risk graph, layers of protection analysis (LOPA), risk matrix as well as quantitative risk analysis. In this paper, the way from the identified risk to a safety concept is demonstrated.

KEYWORDS Risk / Safety Concept 
VOLKER ARNDT, BASF SE

W

ie sieht Risiko praktisch aus? Im Bereich Sicherheit wird es als Anzahl Tote pro Jahr ausgedrückt. Um zu entscheiden, ob ein Risiko zu hoch ist, braucht man Akzeptanzkriterien. Hierzu gibt es international verschiedene Ansätze, die aber meist in einem ähnlichen Bereich liegen. Bild 1 zeigt als Beispiel die Akzeptanzkriterien von Großbritannien [1]. Dabei werden drei Bereiche unterschieden. Ein Risiko, das kleiner als $10^{-6}$ Tote pro Jahr ist, wird als allgemein akzeptabel angesehen (grün). Bei der Frage, was nicht akzeptabel ist, unterscheidet die britische Regierung zwischen dem Risiko am Arbeitsplatz und dem Risiko für die Öffentlichkeit. Die Werte liegen bei $10^{-3}$ beziehungsweise $10^{-4}$ Toten pro Jahr und stellen das Grenzrisiko dar. Ein Risiko, das größer ist, wird nicht akzeptiert (rot). Zwischen diesen beiden Bereichen liegt ein Sektor, in dem das Risiko als tolerierbar gilt (gelb). Die Bilder 2 und 3 veranschaulichen diese Zahlen.

In Bild 2 ist die Entwicklung für das Risiko in verschiedenen Branchen der deutschen Industrie über 50 Jahre dargestellt. Die Zahlen stammen aus Veröffentlichungen der Berufsgenossenschaften [2,3]. Drei Aspekte zeichnen sich ab:

1 | In den 60er-Jahren lagen viele Branchen selbst als Durchschnitt an der oberen Grenze der aufgezeigten Akzeptanzwerte.

2 | Alle Branchen haben sich über die letzten 50 Jahre ständig verbessert.

3 | Die chemische Industrie (die schwarzen Punkte) gehört zu den sichersten Branchen.

Bild 3 enthält einige Beispiele aus dem Alltag [1, 2, 3]. Für Risiken, bei denen das Risiko per Einzeltätigkeit angegeben war, wurde zur besseren Vergleichbarkeit eine typische Anzahl der Tätigkeit pro Jahr angenommen (Zahl in Klammer angegeben) und das entstehende Risiko pro Jahr eingetragen.

- Beispiele verschiedener Hobbys

- Häuslicher Gasanschluss (in UK)
- Teilnahme am Verkehr (Straße in Deutschland,

Bahn in UK, Urlaubsreise mit dem Flugzeug)

- Blitzschlag bei Gewitter

- Erkrankung an Krebs

- Branche Chemie in Deutschland

Wenn man sieht, dass einige hohe Risiken im Alltag leicht akzeptiert werden, während manche niedrige Risiken Befürchtungen auslösen, stellt sich die Frage, ob Risiko überhaupt objektiv ist. Die Antwort ist eindeutig Nein. Hierfür gibt es drei Gründe:

Zum Einen kann ein positives Feedback die Risikowahrnehmung zurückdrängen. So wird das objektiv hohe und bekannte Krebsrisiko beim Rauchen durch den Genussgewinn kompensiert und verdrängt.

Außerdem werden freiwillig eingegangene Risiken eher akzeptiert als aufgezwungene Risiken, wie an vielen Freizeitaktivitäten erkennbar.

Schließlich werden seltene Großereignisse stärker wahrgenommen und prägen daher die Risikowahrnehmung intensiver als häufige kleine Ereignisse. Mathematisch ist 1 mal 100 gleich 100 mal 1, aber ein Bahnunglück oder Flugzeugabsturz wird anders aufgenommen als viele Autounfälle.

\section{ENTWICKLUNG DES SICHERHEITSKONZEPTS}

Der erste Schritt auf dem Weg zum Sicherheitskonzept ist die Identifikation von Szenarien. Die Grafik in Bild 4 ist dem EU-Projekt ARAMIS [4] entnommen und stellt die Elemente eines Szenarios dar. Im Zentrum eines Szenarios liegt immer eine Stoff- oder Energiefreisetzung - kritisches Ereignis genannt. Auf der linken Seite sieht man alle Gründe, die zu dem Ereignis führen können. Die Darstellung der „Und“- und „Oder“-Verknüpfungen wird als Fehlerbaum bezeichnet. Auf der rechten Seite zeigt der Ereignisbaum die Varianten, in denen eine Freisetzung Schaden anrichtet. Man kann nun einzelne Wege durch Barrieren unterbrechen. Auf der linken Seite sind sie eintrittsverhindernd und auf der rechten Seite schadensbe- 
grenzend. Die Barrieren senken das Risiko, indem sie entweder die Eintrittshäufigkeit senken oder die Auswirkung verringern. Das Maß dieser Risikosenkung hängt dabei direkt von der Zuverlässigkeit der Barriere ab. Schutzmaßnahmen sind Barrieren mit besonders hoher Zuverlässigkeit. Meist ist es sinnvoll, mehrere Barrieren nach dem Zwiebelschalenprinzip zu kombinieren.

Ein Schwerpunkt im Sicherheitskonzept sind die Schutzmaßnahmen. Hier gibt es meist verschiedene Al- ternativen. In der Regel wird in dieser Prioritätsreihenfolge vorgegangen:

1| Inhärente Sicherheit

2 | Eigensicheres Design

3| Mechanische Schutzeinrichtungen

4 | PLT-Schutzeinrichtungen

5 | Organisatorische Schutzmaßnahmen
BILD 1:

Risikogrenzwerte in Großbritannien

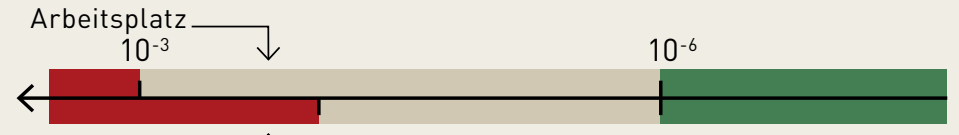

$$
\text { Öffentlichkeit- } \uparrow \overbrace{}^{10^{-4}}
$$

Nicht akzeptabel

Tolerierbar Grenzrisiko
Allgemein akzeptabel

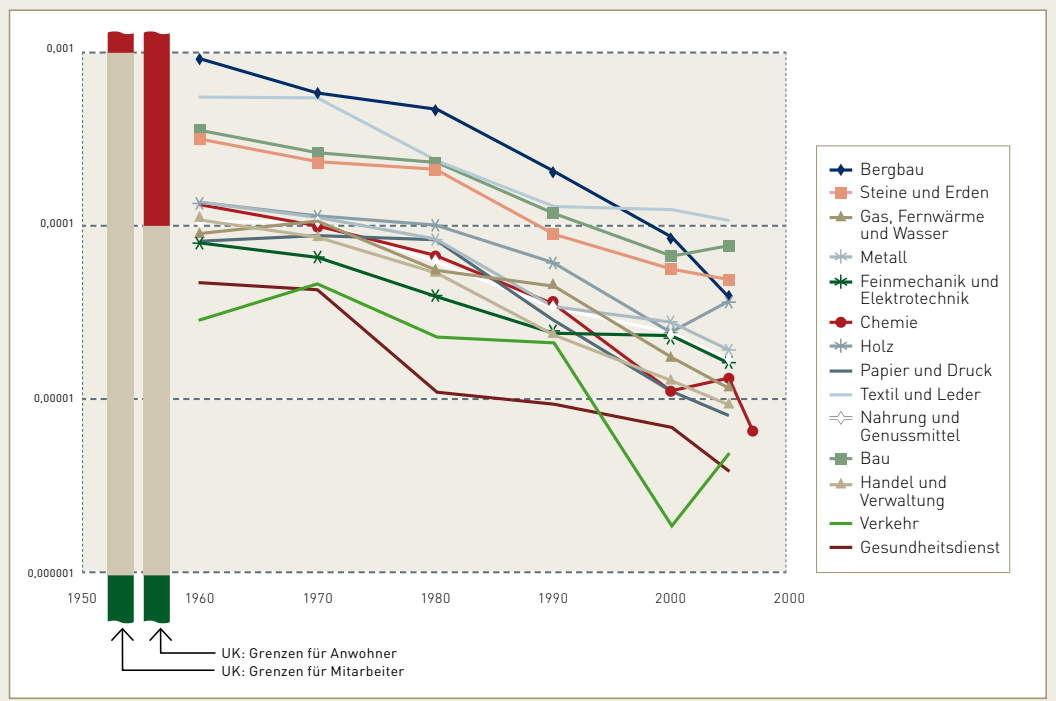

BILD 2: Entwicklung des Arbeitsplatzrisikos in Deutschland
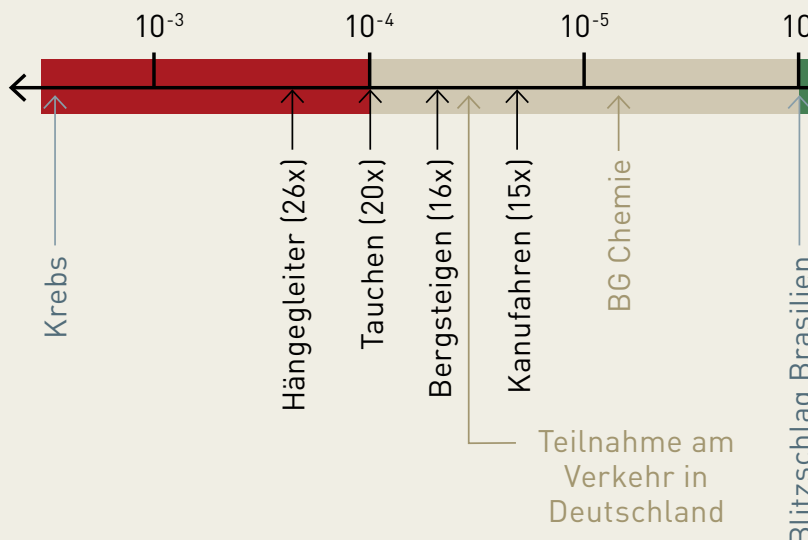

$10^{-6}$ $10^{-7}$

BILD 3: Typische Freizeit- und Naturrisiken

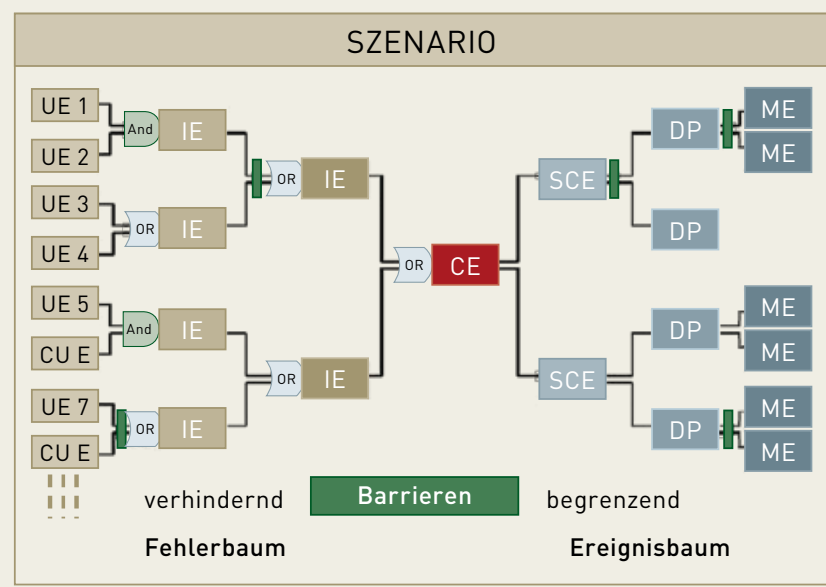

BILD 4: Darstellung eines Szenarios 
Wie schon erwähnt, werden nach dem Zwiebelschalenprinzip zusätzlich schadensbegrenzende Maßnahmen vorgesehen. Wie stellt sich die Risikoreduktion bildlich dar? Dies verdeutlicht Bild 5. Das prozessspezifische Rohrisiko ist das Risiko ohne Gegenmaßnahmen. Vom Rohrisiko muss mit Hilfe der Schutzmaßnahmen mindestens der tolerierbare Bereich erreicht werden. Mit weiteren Maßnahmen nach dem Zwiebelschalenprinzip wird das Risiko dann in den akzeptablen Bereich zum verbleibenden Risiko gebracht.

\section{WERKZEUGE}

Um zu entscheiden, ob Schutzmaßnahmen notwendig sind und welche Qualität sie brauchen, gibt es verschiedene Werkzeuge.

Das erste sind deterministische Methoden ohne Risikobezug. Diese können aus Regelwerken kommen (zum Beispiel Mindestanforderungen an Überfüllschutz bei wassergefährdenden Stoffen) oder per Konvention festgelegt sein. Sie sind einfach anzuwenden und meist etwas konservativ, da die Mehrzahl aller Fälle abgedeckt werden muss. Situationen mit sehr hohem inhärenten Potenzial werden dabei allerdings oft unterschätzt.

Das zweite Werkzeug ist der altbekannte Risikograph. Auch er ist einfach anzuwenden. Allerdings sind die Begriffe unbestimmt und müssen kalibriert werden. Auch hier gibt es Situationen, für die er nicht geeignet ist.

Etwas neuer ist das dritte Werkzeug, die Layers-ofProtection-Analyse (LOPA). Sie scheint einfach anzuwenden zu sein, birgt aber einige Fallstricke. Zuerst muss das inhärente Potenzial bestimmt werden, um die Anzahl der notwendigen „Layers“ festlegen zu können. Dann ist es unbedingt notwendig, dass die Unabhängigkeit einzelner Layers gewährleistet sein muss. Hier ist nach Literaturangaben ein Großteil der LOPAs fehlerhaft, denn LOPA verführt dazu, abhängige Dinge mehrfach zu zählen. Bei richtiger Anwendung, die dann viel aufwändiger ist, sind die Ergebnisse jedoch korrekt.

Das vierte Werkzeug ist die Risikomatrix. Sie ist einfach anzuwenden. Es ist aber eine firmenspezifische, an die jeweiligen Rahmenbedingungen und die Vorgehens- weise angepasste Kalibrierung notwendig. Dann liefert sie robuste Ergebnisse.

Das letzte Werkzeug ist die quantitative Risikoanalyse QRA. Sie ist sehr aufwendig, da sehr viele Szenarien gerechnet werden müssen. Man erhält eine auf mehrere Stellen hinter dem Komma scheinbar sehr präzise Zahl. Auf Grund der notwendigen Vereinfachungen und Annahmen ist dies allerdings ein Trugschluss. So sind die Verteilungsfunktionen für die Häufigkeiten meist nicht bekannt, und aus der unendlichen Vielfalt an Szenarien muss ein repräsentativer Satz ausgewählt werden. Notwendige bedingte Wahrscheinlichkeiten (beispielsweise Zündung ja oder nein) werden auch bei der QRA per Konvention festgelegt.

\section{DOKUMENTATION}

Nachdem mit einem dieser Werkzeuge festgestellt wurde, dass eine Schutzmaßnahme nötig ist, muss für diese eine Spezifikation geschrieben werden. Die Spezifikation muss nicht nur die offensichtlichen Anforderungen enthalten Was löst aus? Welche Aktion soll erfolgen? Notwendiger SIL? - sondern alle Angaben, die für die Erfüllung der Aufgabenstellung notwendig sind (zum Beispiel Schließzeiten, Leckageanforderungen, angestrebter Prüfmodus, und so weiter).

Die Spezifikation ist nur ein Teil der Dokumentation. Es sollte auch das Sicherheitskonzept als Positivdokumentation beschrieben werden. Ebenso wichtig ist die Dokumentation der Abnahmen, der Prüfvorschrift und der Prüf- und Wartungshistorie zur Aufrechterhaltung der Zuverlässigkeit über den Lebenszyklus.

\section{FAZIT}

Das Risiko ist die Kombination aus Eintrittshäufigkeit und Schadensausmaß. Barrieren senken die Eintrittshäufigkeit oder das Schadensausmaß. Das Sicherheitskonzept erhält man durch die Beschreibung aller Barrieren. Das Sicherheitskonzept muss vollständig dokumentiert werden. Spezifikation und Lebenszyklus müssen bei PLT-Schutzeinrichtungen dokumentiert werden.

MANUSKRIPTEINGANG 14.12.2010

\section{REFERENZEN}

[1] Reducing risks, protecting people, HSE's decisionmaking process, Health \& Safety Executive, 2001

[2] BG Statistiken für die Praxis 2006, Deutsche Gesetzliche Unfallversicherung e.V. (DGUV)

[3] Jahresbericht der BG Chemie 2007, BG Chemie

[4] ARAMIS, Accidental Risk Assessment Methodology for Industries in the context of the Seveso II Directive - WP 1 Methodology for the Identification of Major Accident Hazards, Draft report version 1, September 2002

\section{AUTOREN}

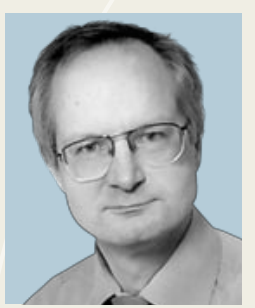

DR. VOLKER ARNDT (geb. 1956) ist DiplomChemiker. Nach Tätigkeiten bei BASF in der Forschung, in der Betriebsleitung verschiedener Betriebe und mehrjähriger Leitung der Anlagensicherheit in Nordamerika führt er seit 2000 die Gruppe Anlagensicherheit B der BASF.

BASF SE,

GUS/AB - M 940, Carl-Bosch-Straße 38, D-67056 Ludwigshafen, Tel. +49 (0) 62160791 13, E-Mail: volker.arndtabasf.com 